\title{
Thymoquinone inhibits metastatic phenotype and epithelial-mesenchymal transition in renal cell carcinoma by regulating the LKB1/AMPK signaling pathway
}

\author{
BO KOU ${ }^{1 *}$, QINGSHAN KOU ${ }^{2 *}$, BO MA $^{3^{*}}$, JING ZHANG $^{4}$, BORUI SUN ${ }^{5}$, \\ YANG YANG $^{1}$, JIANPENG LI ${ }^{1}$, JINSONG ZHOU ${ }^{6,7}$ and WEI LIU ${ }^{8}$
}

\begin{abstract}
${ }^{1}$ Department of Cardiovascular Surgery, The First Affiliated Hospital of Medical School, Xi'an Jiaotong University, Xi'an, Shaanxi 710061; ${ }^{2}$ Medical Center, The First People's Hospital of Xianyang, Xianyang, Shaanxi 712000;

${ }^{3}$ Department of Ophthalmology, The First Affiliated Hospital of Medical School, Xi'an Jiaotong University; ${ }^{4}$ Department of Gynaecology, Shaanxi Provincial People's Hospital; ${ }^{5}$ Department of Anesthesiology, The First Affiliated Hospital of Medical School, Xi'an Jiaotong University; ${ }^{6}$ Department of Human Anatomy, Histology and Embryology, School of Basic Medical Sciences, Xi'an Jiaotong University Health Science Center; ${ }^{7}$ Key Laboratory of Environment and Genes Related to Diseases, Xi'an Jiaotong University, Ministry of Education of China; ${ }^{8}$ Department of Vascular Surgery,

The First Affiliated Hospital of Medical School, Xi'an Jiaotong University, Xi'an, Shaanxi 710061, P.R. China
\end{abstract}

Received May 6, 2017; Accepted May 10, 2018

DOI: $10.3892 / o r .2018 .6519$

\begin{abstract}
Thymoquinone, isolated from the seeds of Nigella sativa, has exhibited antitumor properties in a variety of cancer types. However, few studies have investigated the effect of thymoquinone (TQ) on migration and invasion in renal cell carcinoma (RCC). In the present study, our results confirmed that TQ significantly inhibited the migration and invasion of the human RCC 769-P and 786-O cell lines, as demonstrated by wound healing and Transwell assays. Additionally, TQ upregulated the expression of E-cadherin and downregulated the expression of Snail, ZEB1 and vimentin at the mRNA and protein levels in a concentration-dependent manner. Subsequently, the phosphorylation levels of liver kinase B1 (LKB1) and AMP-activated protein kinase (AMPK) were increased upon TQ treatment. To further validate the role of LKB1/AMPK signaling, we revealed that TQ-mediated
\end{abstract}

Correspondence to: Professor Jinsong Zhou, Department of Human Anatomy, Histology and Embryology, School of Basic Medical Sciences, Xi'an Jiaotong University Health Science Center, 76 West Yanta Road, Xi'an, Shaanxi 710061, P.R. China E-mail: 492526094@qq.com

Dr Wei Liu, Department of Vascular Surgery, The First Affiliated Hospital of Medical School, Xi'an Jiaotong University, 277 West Yanta Road, Xi'an, Shaanxi 710061, P.R. China

E-mail: zjs301@mail.xjtu.edu.cn; lizhang1988617@163.com

*Contributed equally

Key words: thymoquinone, renal cell carcinoma, LKB1, AMPK, epithelial-mesenchymal transition increase of E-cadherin level and reduction of Snail level could be further enhanced by LKB1 overexpression. Furthermore, co-treatment with the AMPK inhibitor Compound $\mathrm{C}$ attenuated the anti-metastatic effect of TQ on RCC and partially abrogated the high expression of E-cadherin and the low expression of Snail mediated by TQ. In contrast, the AMPK activator AICAR demonstrated the opposite effect. Collectively, the present study revealed that TQ could markedly suppress the metastatic phenotype and reverse the epithelial-mesenchymal transition in RCC by regulating the LKB1/AMPK signaling pathway, indicating that TQ may be a potential therapeutic candidate against RCC.

\section{Introduction}

Renal cell carcinoma (RCC) is the most common type of kidney cancer worldwide (1). Despite the improvement of diagnostic techniques and therapeutic treatments, the majority of patients with RCC are diagnosed at an advanced stage or have already presented with metastasis. It is reported that in $30 \%$ of patients with RCC, metastatic lesions have already developed at the stage of diagnosis (2). In view of the poor response of patients with RCC to chemotherapy and radiotherapy, the treatment of metastatic unresectable RCC consists a great challenge for clinicians. Therefore, it is essential to find potential therapeutic agents for the treatment of RCC.

Thymoquinone (TQ), isolated from the seeds of Nigella sativa, is a natural polyphenolic compound (3). Numerous studies have reported that TQ has broad pharmacological effects. For example, TQ is regarded as an IRAK1 (interleukin receptor-associated kinase 1) inhibitor with anti-inflammatory activities (4). In addition, TQ is reported to have antioxidant effects in activated BV-2 murine microglial cells (5). Additionally, previous studies revealed that TQ has 
antitumor activities such as suppression of proliferation, induction of apoptosis, inhibition of metastasis and enhancement of chemosensitivity (6-9). In RCC, TQ has been found to induce apoptosis by downregulating c-FLIP and Bcl-2 (10). However, few studies have been performed about the effect of TQ on migration and invasion in RCC.

Liver kinase B1 (LKB1), also known as serine/threonine kinase 11 (STK11), was first identified in Peutz-Jeghers syndrome (11). Studies revealed that LKB1 has been verified to regulate cell polarity and maintain energy balance $(12,13)$. Additionally, it is well known that LKB1 directly phosphorylates the AMP-activated protein kinase (AMPK) at the Thr172 site. Accumulating evidence indicated that the role of LKB1 in tumor progression is vital and that the LKB1/AMPK pathway participated in the migratory and invasive process of various tumors, including colon, breast and lung cancer (14-16).

In the present study, we aimed to explore the correlation between TQ and metastasis in RCC and the underlying function mechanism of TQ against RCC.

\section{Materials and methods}

Reagents. TQ was obtained from Sigma-Aldrich (St. Louis, MO, USA) and dissolved in dimethyl sulfoxide (DMSO). Primary rabbit monoclonal antibodies (diluted at 1:1,000) against phosphorylated-LKB1 (3482), LKB1 (3050), phosphorylated-AMPK (9957), AMPK (9957), E-cadherin (3195), Snail (3879), ZEB1 (3396), vimentin (5741) and $\beta$-actin (4970) were purchased from Cell Signaling Technology, Inc. (Beverly, MA, USA). Furthermore, 3-(4,5-dimethylthiazol-2-yl)-2,5-diphenyltetrazolium bromide (MTT), the AMPK inhibitor Compound $\mathrm{C}$ (ComC) and the AMPK activator AICAR were obtained from Sigma-Aldrich.

Cell culture. HK2, a human renal tubular epithelial cell line and the human RCC cell lines 769-P and 786-O were obtained from the American Type Culture Collection (ATCC, Manassas, VA, USA). These three cell lines were grown in RPMI-1640 medium supplemented with $10 \%$ fetal bovine serum (FBS; Gibco, Grand Island, NY, USA), $100 \mu \mathrm{g} / \mathrm{ml}$ streptomycin and $100 \mathrm{U} / \mathrm{ml}$ penicillin (Invitrogen, Carlsbad, CA, USA). All cells were cultured at $37^{\circ} \mathrm{C}$ in a humidified incubator with $5 \% \mathrm{CO}_{2}$ atmosphere.

Cell proliferation assay. A modified MTT assay was used to detect the growth inhibition of TQ on RCC. Briefly, the 769-P and 786-O cells were seeded in 96-well plates with $\sim 90 \%$ density and treated with ascending concentrations of TQ $(0.5,1,2.5,5,10,15$ and $20 \mu \mathrm{M})$ at different time-points $(0,24$, 48 and $72 \mathrm{~h}$ ). Subsequenlty, each well was mixed with $0.5 \mathrm{mg} / \mathrm{ml}$ MTT dye solution for another $4 \mathrm{~h}$ at $37^{\circ} \mathrm{C}$. Subsequently, the culture medium was removed and $150 \mu l$ dimethyl sulfoxide (DMSO) was added to dissolve the formazan crystals. The optical density (OD) of each well was determined at $490 \mathrm{~nm}$ by a 96-well microplate reader (Bio-Rad Laboratories, Hercules, CA, USA). The inhibitory rate of cell growth was calculated as follows: [(OD 490 control group - OD $490_{\text {treated group }}$ /OD $490_{\text {control }}$ group] $\mathrm{x} 100$.

Wound healing assay. The RCC 769-P and 786-O cell lines were seeded onto 6-well plates. When the cell density reached up $90-100 \%$, scratch wounds were created across the monolayer with the tip of a 200- $\mu$ l pipette. Subsequently the wounded cultures were incubated in a serum-free medium upon TQ treatment at 0 and $24 \mathrm{~h}$ and images (magnification x100) were captured by an inverted microscope to evaluate the migratory property. The experiments were performed in triplicate.

Transwell migration assay. Transwell migration assay was used to assess the effect of TQ on RCC cell migration. The

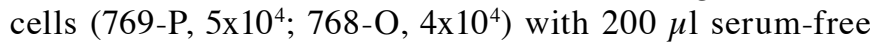
medium were seeded into the upper chamber, while $10 \%$ fetal calf serum-containing medium was added to the lower chamber. Twenty-four hours later, the migrated cells on the bottom of the filter were fixed with $4 \%$ paraformaldehyde, followed by $0.1 \%$ crystal violet staining (Beyotime Institute of Biotechnology, Shanghai, China). The cells were then counted in five independent visual fields using an optical microscope (Olympus Corp., Tokyo, Japan) at a magnification $\mathrm{x} 100$.

Matrigel invasion assay. The effect of TQ on the invasiveness of the RCC cells was detected by a Matrigel invasion assay using a Millicell chamber (Millipore, Billerica, MA, USA). Fifty microliters of mixture (Matrigel, serum-free medium, 1:5) were seeded onto the top chamber for $5 \mathrm{~h}$. Subsequently, the cells (769-P, 10x104; 768-O, 8x10 $)$ in $200 \mu 1$ serum-free medium were treated with TQ for $24 \mathrm{~h}$ following the instructions of the Transwell migration assay.

Quantitative real-time PCR assay. Following the treatment of the 769-P and 786-O cell lines with ascending concentrations of TQ $(2.5,5$ and $10 \mu \mathrm{M})$, their total RNA was extracted using TRIzol reagent (Invitrogen). Complementary DNA (cDNA) was then synthesized using a PrimerScript RT reagent kit (Takara, Dalian, China). Then the relative levels of target gene messenger RNA (mRNA) were evaluated by quantitative real-time PCR assay (qRT-PCR) using FAST SYBR Green Master Mix. The primers are as follows: Human E-cadherin (119 bp) forward, 5'-CGAGAGCTACACGTTCACGG-3' and reverse, 5'-GGGTGTCGAGGGAAAAATAGG-3'; human Snail (140 bp) forward, 5'-TCGGAAGCCTAACTACAG CGA-3' and reverse, 5'-AGATGAGCATTGGCAGCGAG-3'; human ZEB1 (86 bp) forward, 5'-GATGATGAATGCGAG TCAGATGC-3' and reverse, 5'-ACAGCAGTGTCTTGTTGT TGT-3'; human vimentin (238 bp) forward, 5'-GACGCCATC AACACCGAGTT-3' and reverse, 5'-CTTTGTCGTTGGTTA GCTGGT-3'; human $\beta$-actin (250 bp) forward, 5'-CATGTA CGTTGCTATCCAGGC-3' and reverse, 5'-CTCCTTAAT GTCACGCACGAT-3'. The n-fold change in the expression of mRNA was analyzed according to the $2^{-\Delta \Delta C t}$ method.

Western blotting. Briefly, the RCC 769-P and 786-O cells were harvested after certain treatment and lysed on ice for $10 \mathrm{~min}$ in a lysis buffer $[10 \mathrm{mmol} / 1$ Tris- $\mathrm{HCl}(\mathrm{pH} 7.4), 150 \mathrm{mmol} / \mathrm{l}$ $\mathrm{NaCl}, 0.1 \%$ sodium dodecyl sulfate (SDS), $1 \mathrm{mmol} / 1$ ethylenediaminetetraacetic acid, $1 \mathrm{mmol} / 1$ ethylene glycol tetraacetic acid, $0.3 \mathrm{mmol} / 1$ phenylmethylsulfonyl fluoride, $0.2 \mathrm{mmol} / 1$ sodium orthovanadate, $1 \% \mathrm{NP}-40,10 \mathrm{mg} / \mathrm{ml}$ leupeptin and $10 \mathrm{mg} / \mathrm{ml}$ aprotinin]. Subsequently, the clarified protein lysates (about $40-60 \mu \mathrm{g}$ ) were separated by 10 or $15 \%$ sodium dodecyl 
A<smiles>CC1=CC(=O)C(C(C)C)=CC1=O</smiles>

C

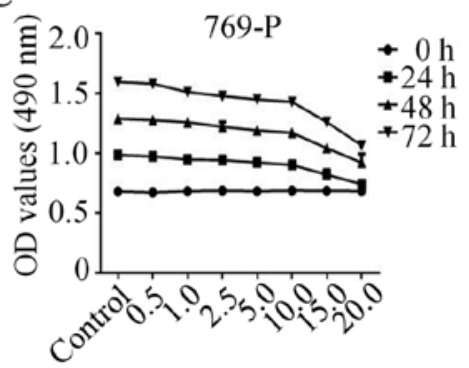

Thymoquinone treatment $(\mu \mathrm{M})$

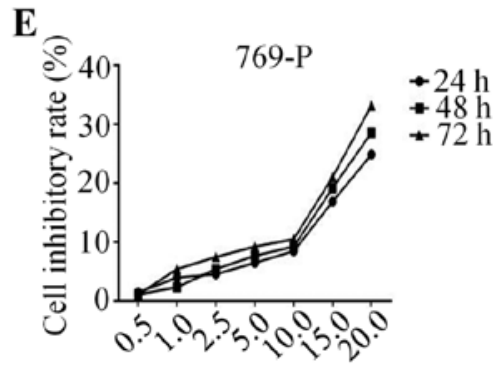

Thymoquinone treatment $(\mu \mathrm{M})$

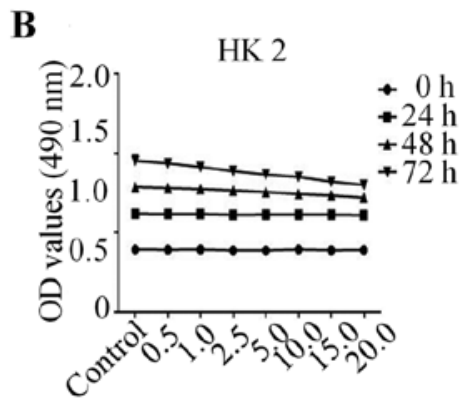

Thymoquinone treatment $(\mu \mathrm{M})$
D

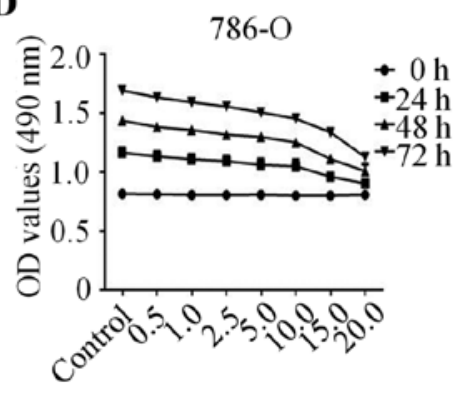

Thymoquinone treatment $(\mu \mathrm{M})$
$\mathbf{F}$

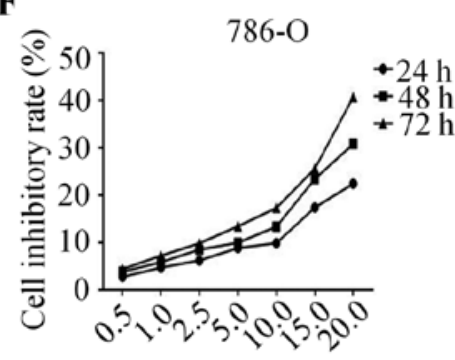

Thymoquinone treatment $(\mu \mathrm{M})$

Figure 1. TQ inhibits the cell growth of human RCC cells. (A) The chemical structure of TQ. (B) The cytotoxic effect of TQ on normal renal tubular epithelial HK2 cell line. After the 769-P and 786-O cells with 90\% density were treated with negative control or various doses of TQ $(0.5,1.0,2.5,5.0,10,15,20 \mu \mathrm{M})$ for different time-points $(0,24,48$ and $72 \mathrm{~h})$, the viability of these two renal cell carcinoma cells was detected by a modified MTT assay. The OD and inhibitory rate of TQ in (C and E) 769-P and (D and F) 786-O cells. The values are presented as the mean $\pm \mathrm{SD}$. TQ, thymoquinone; RCC, renal cell carcinoma; OD, optical density.

sulfate-polyacrylamide gel electrophoresis (SDS-PAGE) and transferred onto polyvinylidene difluoride (PVDF) membranes (EMD Millipore, Bedford, MA, USA). Subsequently, the membranes were incubated with antibodies against phosphorylated-LKB1, total-LKB1, phosphorylated-AMPK, AMPK, E-cadherin (E-Ca), Snail and $\beta$-actin overnight at $4^{\circ} \mathrm{C}$. The bands were then washed with TBST (Tris-buffered saline with Tween) buffer and incubated with horseradish peroxidase (HRP)-linked secondary antibody at room temperature $\left(25^{\circ} \mathrm{C}\right)$ for $1 \mathrm{~h}$. Finally, the protein bands were detected by an enhanced chemiluminescence detection kit (Bio-Rad Laboratories) and exposed to Image Lab 4.0 (Bio-Rad Laboratories) imaging software.

Plasmid transfection. LKB1 cDNA was cloned into pcDNA3.1 vector. The cells were seeded onto 6-well plates and transfected with the corresponding plasmid using X-tremeGENE HP DNA Transfection Reagent (Roche, Basel, Switzerland) according to the manufacturer's instructions.

Statistical analysis. All experimental data are presented as the means \pm standard deviation (SD) and GraphPad Prism (GraphPad Software, Inc., San Diego, CA, USA) software was used for statistical analyses. Differences between two groups were analyzed using Student's t-test (two-sided), while one-way ANOVA test was used for comparisons among multiple independent groups. $\mathrm{P}<0.05$ was considered to indicate a statistically significant difference.

\section{Results}

The anti-proliferative effect of TQ on RCC cells. The chemical structure of TQ is depicted in Fig. 1A. Firstly, we detected the effect of TQ on the normal renal tubular epithelial HK2 cell line. The results demonstrated that there was no significant change in cell growth upon TQ treatment for $24 \mathrm{~h}$, while a slight decrease in cell growth with TQ treatment for 48 and $72 \mathrm{~h}$ was observed, indicating a low cytotoxic effect of TQ on nomal epithelial cells (Fig. 1B). Subsequently, in order to confirm the effect of TQ on RCC cell migration and invasion, it was essential to determine the concentration-dependent effect of TQ on cell viability. Human RCC 769-P and 786-O cells with $90 \%$ density were exposed to TQ treatment $(0.5$, $1,2.5,5,10,15$ and $20 \mu \mathrm{M})$ at different time-points $(0,24$, 48 and $72 \mathrm{~h}$ ), which revealed a gradually decreasing cell proliferation in a concentration- and time-dependent manner (Fig. 1C and D). Lower doses of TQ (up to $10 \mu \mathrm{M}$ ) exhibited a less than $10 \%$ inhibitory rate of cell growth, while higher doses of TQ (beyond $10 \mu \mathrm{M}$ ) exhibited a significant inhibition of cell proliferation (Fig. 1E and F). In view of this, the concentration of $10 \mu \mathrm{M}$ at $24 \mathrm{~h}$ was chosen to explore the anti-metastatic potential of TQ on RCC cells.

The anti-metastatic effect of TQ on RCC cells. A wound healing assay was used to explore the effect of TQ on cell migration. The migration speed of the 769-P cell line was significantly reduced in the presence of TQ compared to the 
A

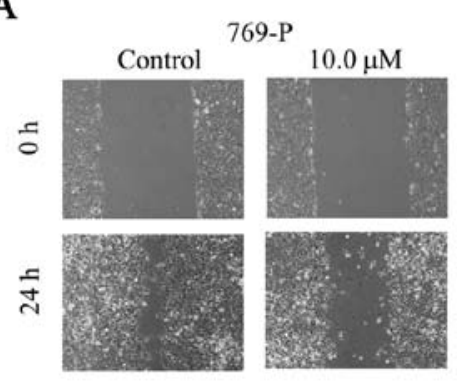

B

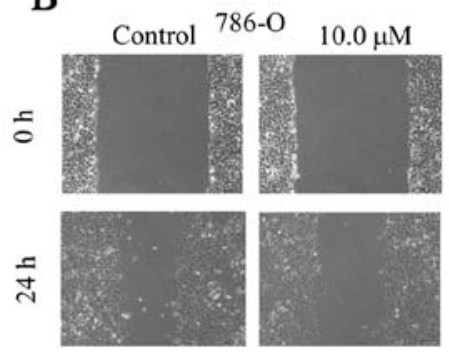

C

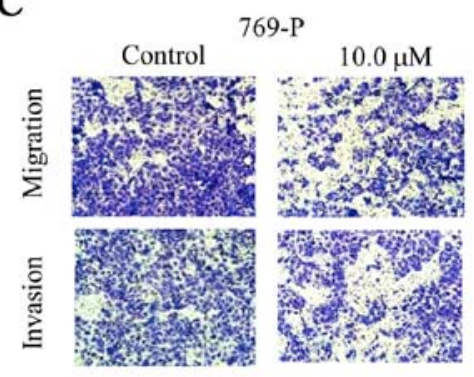

D

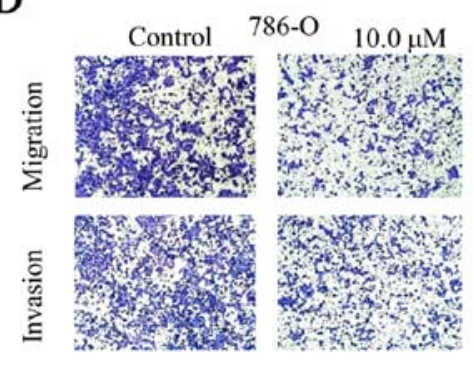

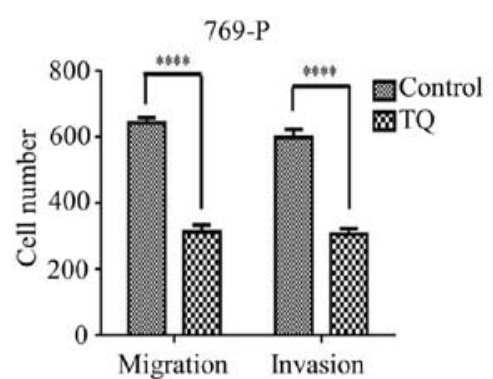

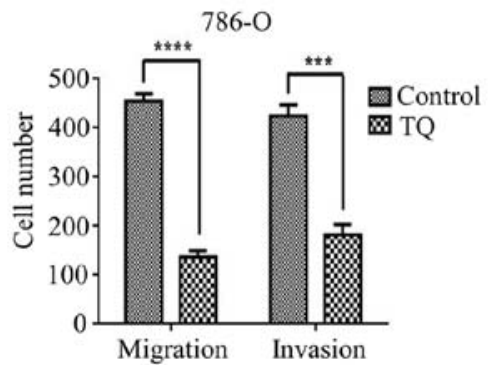

Figure 2. TQ suppresses cell migration and invasion of RCC cells. (A and B) Wound healing assay was used for the detection of inhibition of TQ on 769-P and 786-O cells. The width of scratches was assessed in negative control or TQ $(10 \mu \mathrm{M})$ group in RCC 769-P and 786-O cells. (C and D) Using Transwell migration assay and Matrigel invasion assay, 769-P and 786-O cells were exposed to TQ treatment (10 $\mu \mathrm{M})$ for $24 \mathrm{~h}$ and the number of migrated or invaded cells per chamber was assessed. The experiments were performed in triplicate $\left({ }^{* * *} \mathrm{P}<0.001\right.$ and $\left.{ }^{* * * * *} \mathrm{P}<0.0001\right)$. TQ, thymoquinone; RCC, renal cell carcinoma.

untreated cells (Fig. 2A). Similar results were observed in the TQ-treated 786-O cell lines (Fig. 2B). Subsequently, the RCC 769-P and 786-O cell lines were treated with TQ for the Transwell migration assay and a significant reduction in cell migration was observed under the TQ treatment compared with that in the untreated group. The number of migrated cells on the lower surface of the chamber is demonstrated in Fig. 2C and D. The results indicated that TQ plays a crucial role in inhibiting the migration of RCC cells.

Subsequently, to confirm the inhibitory effect of TQ on the invasion of 769-P and 786-O cells, a Matrigel invasion assay was used for the detection of the cell invasion ability. After treatment with $10 \mu \mathrm{M}$ TQ for $24 \mathrm{~h}$, the invasiveness of the 769-P cell line was significantly inhibited compared with the negative control group (Fig. 2C). Similar results were observed in the 786-O cell line (Fig. 2D). Collectively, these data revealed that TQ could suppress the invasion of RCC cells.

The reversal effect of TQ on EMT (epithelial-mesenchymal transition) in $R C C$ cells. It has been widely reported that EMT is closely correlated with metastasis (17). To verify the change of EMT markers upon TQ treatment, we detected the mRNA levels of E-cadherin, Snail, ZEB1 and vimentin at different concentrations of TQ. As expected, the level of E-cadherin was upregulated, while the expression of Snail, ZEB1 and vimentin was downregulated upon TQ treatment in a concentration-dependent manner (Fig. 3A and B). Subsequently, the results of western blot analysis revealed that TQ increased the protein level of E-cadherin, while it reduced the protein levels of Snail, ZEB1 and vimentin in a concentration-dependent pattern (Fig. 3C and D). These results indicated that TQ could reverse EMT in RCC.
The anti-metastatic effect of $T Q$ is mediated by the LKB1/AMPK signaling pathway. A previous study revealed that the LKB1/AMPK signaling is implicated in cancer metastasis (18). Firstly, western blot analyses were performed to evaluate the expression of LKB1 and AMPK. As depicted in Fig. 4A, an increase in the phosphorylation levels of LKB1 and AMPK was observed in the 769-P cell line upon TQ treatment, while the total LKB1 and AMPK exhibited no change under TQ treatment. In addition, following $24 \mathrm{~h}$ of treatment, we observed a concentration-dependent increase in phosphorylated-LKB1 and phosphorylated-AMPK in 786-O cells treated with TQ, which was in accordance with the above-mentioned results (Fig. 4B). The results of western blot analysis demonstrated that TQ significantly induced the phosphorylation of LKB1 and AMPK in RCC cells. To further validate whether LKB1 participated in the inhibitory effect of TQ on 769-P and 786-O cell lines, LKB1 was overexpressed by plasmid transfection. The results revealed that overexpression of LKB1 further enhanced the expression of E-cadherin, while it reduced the expression of Snail in the TQ-treated 769-P and 786-O cell lines (Fig. 4C and D). In addition, phosphorylated-AMPK, the downstream kinase of LKB1, was upregulated under the overexpression of LKB1. Collectively, these results confirmed the role of LKB1 in the regulation of EMT in RCC.

To further explore whether LKB1/AMPK signaling plays a vital role in TQ-inhibited cell migration and invasion, Compound C (ComC, AMPK inhibitor) and AICAR (AMPK activator) were used in combination with TQ for the subsequent experiment. The findings revealed that co-treatment with ComC attenuated the anti-metastatic effect of TQ on 769-P and 786-O cells, as revealed by the Transwell migration assay and the Matrigel invasion assay (Fig. 5A and B). Additionally, 
A



C

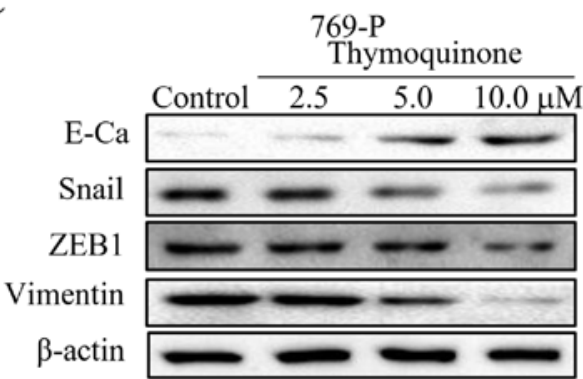

B

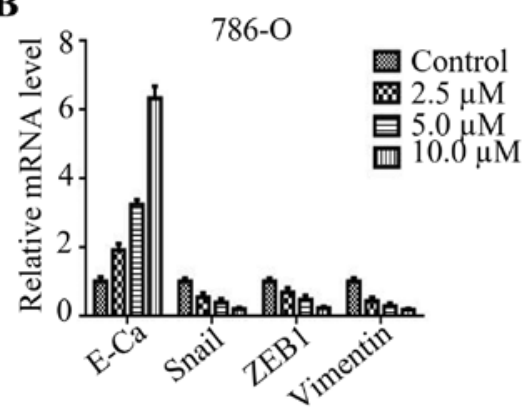

D



Figure 3. TQ markedly reverses EMT in RCC cells. (A and B) Quantitative real-time PCR was used to explore the expression of E-cadherin, Snail, ZEB1 and vimentin in 769-O and 786-O cell lines upon thymoquinone treatment $(2.5,5.0$ and $10 \mu \mathrm{M})$. (C and D) 769-O and 786-O cells treated with certain doses of TQ $(2.5,5.0$ and $10 \mu \mathrm{M})$ were subjected to western blotting for E-cadherin (E-Ca), Snail, ZEB1, vimentin and $\beta$-actin. Representative protein bands from three experiments are shown. TQ, thymoquinone; EMT, epithelial-mesenchymal transition; RCC, renal cell carcinoma.

A

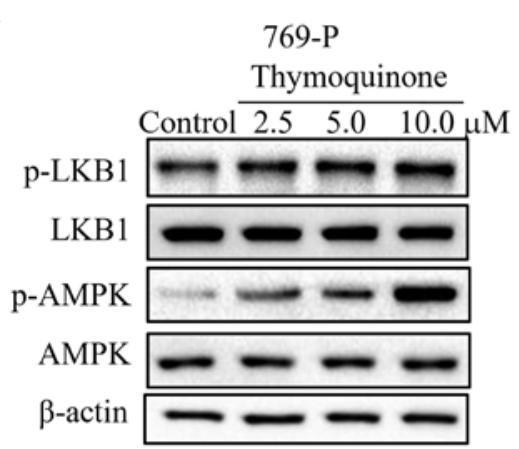

C

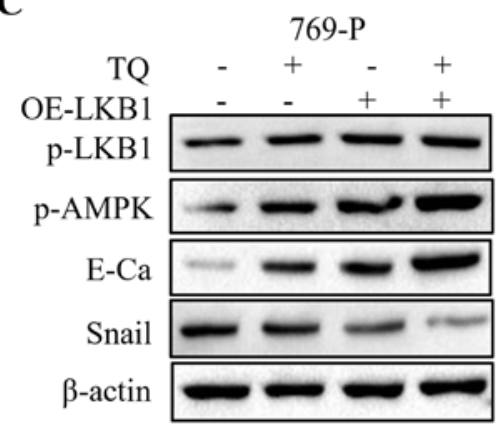

B

786-O

Thymoquinone

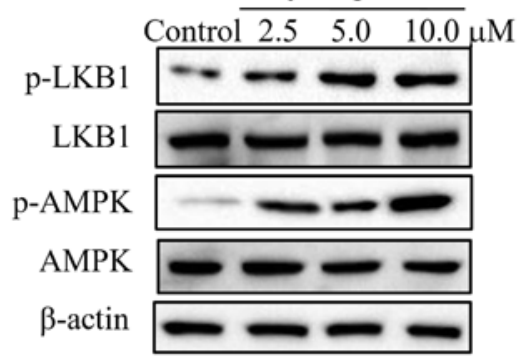

D

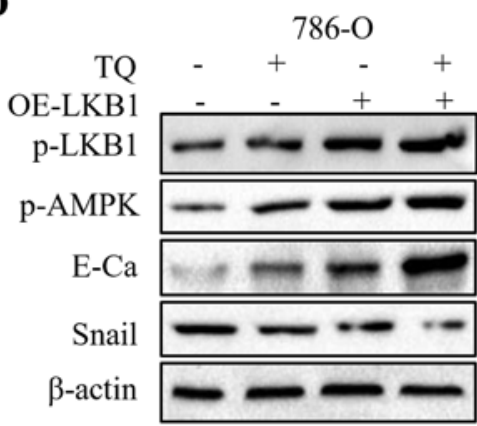

Figure 4. TQ reduces the expression of LKB1 and AMPK in RCC cells. (A and B) 769-O and 786-O cells treated with certain doses of TQ (2.5, 5.0 and $10 \mu \mathrm{M})$ were subjected to western blotting for phosphorylated-LKB1, LKB1, phosphorylated-AMPK, AMPK and $\beta$-actin. Representative protein bands from three experiments are shown. (C and D) Cells overexpressing LKB1 by plasmid transfection were synergistically treated with TQ to detect the change of EMT markers in 769-P and 786-O cells. Western blot analysis was used to analyze the expression of phosphorylated-LKB1, phosphorylated-AMPK, E-cadherin, Snail and $\beta$-actin. Representative results from three independent experiments are shown. RCC, renal cell carcinoma; EMT, epithelial-mesenchymal transition; OE, overexpressing.

TQ-mediated upregulation of E-cadherin and downregulation of Snail were partially abolished by the synergistic treatment with ComC (Fig. 5C and D). In contrast, the anti-metastatic effect of TQ on RCC 769-P and 786-O cell lines was further reinforced by AICAR (Fig. 6A and B). In addition, AICAR further strengthened the expression of E-cadherin, while 
A

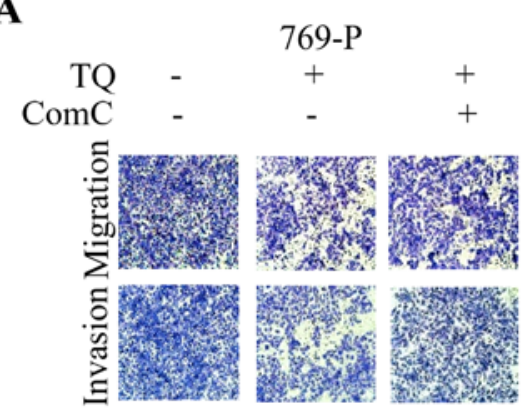

C

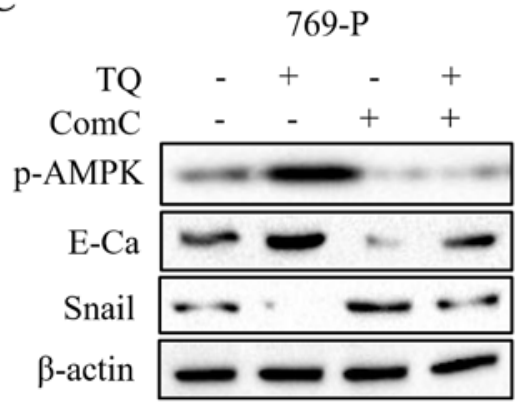

B

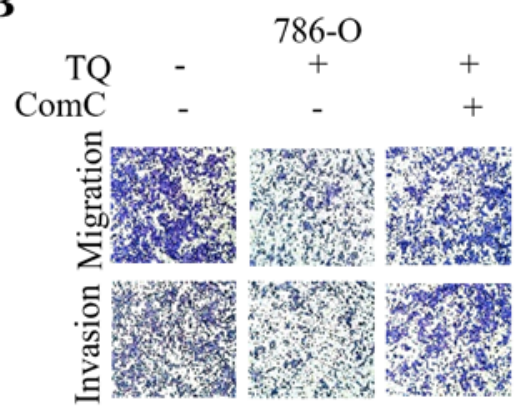

D

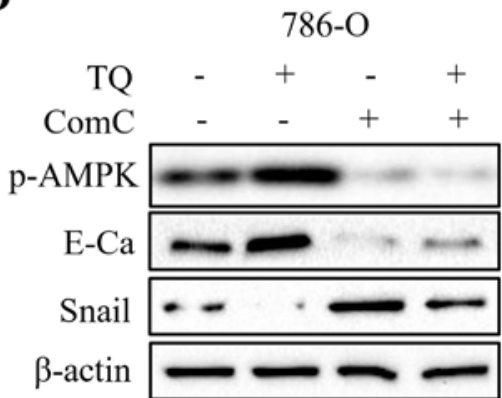

Figure 5. Inactivation of AMPK by Compound C (ComC) attenuates the anti-metastatic effect of TQ on RCC cells. (A and B) Transwell migration assay and Matrigel invasion assay were used to detect the change of cell migration and invasion in 769-P and 786-O cells with different treatments (negative control, $\mathrm{TQ}$, ComC and both TQ and ComC). Five random fields were observed by microscopy. All the experiments were performed in triplicate. (C and D) Western blotting was performed to explore the expression of phosphorylated-AMPK, E-cadherin, Snail and $\beta$-actin in 769-P and 786-O cells with different treatment (negative control, TQ, ComC and both TQ and ComC). Representative bands of three experiments are shown. RCC, renal cell carcinoma; TQ, thymoquinone.

A

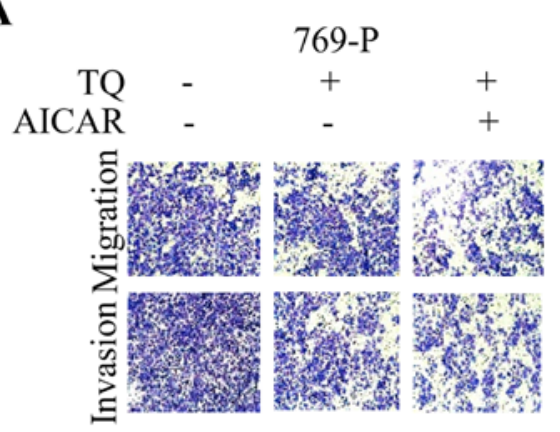

C



B

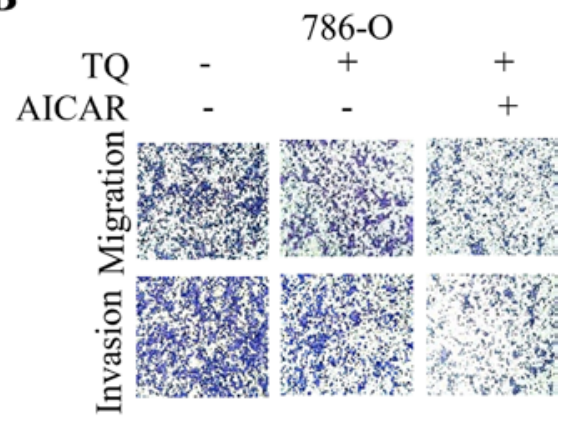

D

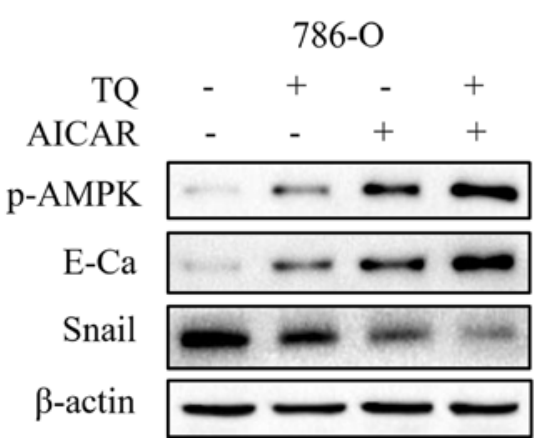

Figure 6. Activation of AMPK by AICAR further decreases migration and invasion, and reverses EMT in TQ-treated RCC cells. (A and B) Using Transwell migration assay and Matrigel invasion assay, the migrated and invaded 769-P and 786-O cells were assessed through different treatments (negative control, TQ, AICAR and both TQ and AICAR). (C and D) 769-P and 786-O cells treated with TQ or AICAR were immunoblotted for phosphorylated-AMPK, E-cadherin, Snail and $\beta$-actin. Representative protein bands from three independent experiments are shown. EMT, epithelial-mesenchymal transition; RCC, renal cell carcinoma; TQ, thymoquinone.

weakened the expression of Snail in 769-P and 786-O cells upon TQ treatment (Fig. 6C and D). These data strongly supported that TQ exhibited anti-metastatic effect through the activation of AMPK phosphorylation. 
In conclusion, these results indicated that the LKB1/AMPK signaling pathway is involved in TQ-regulated migration, invasion and EMT properties in RCC.

\section{Discussion}

It has been widely reported that TQ exerts anti-metastatic activity in various cancers. TQ was found to inhibit the metastasis of melanoma through the downregulation of NLRP3 (NACHT, LRR and pyrin domain-containing protein 3) inflammasome (19). In addition, TQ suppressed the metastatic phenotype of glioblastoma cells, accompanied by the reduction of FAK (focal adhesion kinase), MMP2 (matrix metalloproteinase-2) and MMP9 (20). In the present study, we confirmed that TQ markedly suppressed migration and invasion of human RCC for the first time, as evidenced by the results of the wound healing and Transwell assays. EMT is a complicated process through which cells lose their epithelial properties and tight cell-cell junction, while they gain mesenchymal characteristics. Studies have revealed that EMT is closely correlated with cancer metastasis $(21,22)$. It has been demonstrated that TQ could reverse EMT by reducing the mRNA expression of Twist1 (23). Our findings indicated that TQ drastically increased the expression of E-cadherin, while it decreased the expression of Snail, ZEB1 and vimentin at the mRNA and protein levels, which indicated a strong anti-metastatic activity of TQ on RCC.

A variety of signaling pathways are involved in tumor migration and invasion (24-26). LKB1/AMPK signaling has gained great attention in recent years. Studies indicated that LKB1 deficiency impaired the polarity of mammary epithelial cells, leading to an increase of the migratory and invasive capacity of epithelial cells (27). Furthermore, LKB1 is reported to be a well-known tumor suppressor. It can induce apoptosis and cell cycle arrest, to inhibit tumor progression $(28,29)$. Therefore, dysregulation of LKB1 is closely associated with tumor initiation and progression. The loss of LKB1 was found to upregulate Snail expression, an EMT marker (30). Similarly, ZEB1 was upregulated in LKB1-deficient lung adenocarcinoma cells (31). In the present study, TQ upregulated phosphorylation levels of LKB1 and AMPK in RCC 769-P and 786-O cell lines. In addition, TQ-mediated high expression of E-cadherin and low expression of Snail could be further enhanced by overexpression of LKB1, indicating a critical role of LKB1 in TQ-inhibited EMT of RCC. In addition, AMPK, the downstream of LKB1, is closely associated with tumor growth and neovascularization of cancer cells (32). Furthermore, studies revealed that $\alpha$-enolase promotes metastasis of colorectal cancer by negatively regulating AMPK signaling pathway (33). Our results demonstrated that co-treatment with AMPK inhibitor ComC could impair the anti-metastatic effect of TQ on RCC and reverse TQ-mediated upregulation of E-cadherin and downregulation of Snail. Conversely, the AMPK activator AICAR had the inverse effects.

In conclusion, the present study confirmed that TQ could inhibit metastatic phenotype and reverse EMT in RCC by regulating the LKB1/AMPK signaling pathway. These results indicated that TQ may be an optional therapeutic method for the treatment of RCC. Furthermore, LKB1/AMPK signaling may be a potential therapeutic target against RCC.

\section{Acknowledgements}

Not applicable.

\section{Funding}

The present study was supported in part by a grant from the National Natural Science Foundation of China (no. 81602562), the International Science and Technology Cooperative Project of Shaanxi Province (no. 2017KW-063), the Fundamental Research Funds for the Central University of Xi'an Jiaotong University (no. 1191329722) and the Institutional Scientific Development Foundation of the First Affiliated Hospital of Xi'an Jiaotong University (no. 2015YK17).

\section{Availability of data and materials}

The datasets used during the present study are available from the corresponding author upon reasonable request.

\section{Authors' contributions}

BK, QK and BM conceived and designed the study. BK, JZha, BS and YY performed the experiments and analyzed the data. BK and JL drafted the paper. BK, QK, JL, JZhou and WL drafted, reviewed and edited the manuscript, and were also involved in the conception of the study. All authors read and approved the manuscript and agree to be accountable for all aspects of the research in ensuring that the accuracy or integrity of any part of the work are appropriately investigated and resolved.

\section{Ethics approval and consent to participate}

No applicable.

\section{Patient consent for publication}

Not applicable.

\section{Competing interests}

The authors state that they have no competing interests.

\section{References}

1. Taniguchi H, Ito S, Ueda T, Morioka Y, Kayukawa N, Ueno A, Nakagawa H, Fujihara A, Ushijima S, Kanazawa M, et al: CNPY2 promoted the proliferation of renal cell carcinoma cells and increased the expression of TP53. Biochem Biophys Res Commun 485: 267-271, 2017.

2. Hirata H, Hinoda Y, Nakajima K, Kawamoto K, Kikuno N, Ueno K, Yamamura S, Zaman MS, Khatri G, Chen Y, et al: Wnt antagonist DKK1 acts as a tumor suppressor gene that induces apoptosis and inhibits proliferation in human renal cell carcinoma. Int J Cancer 128: 1793-1803, 2011.

3. Hsu HH, Chen MC, Day CH, Lin YM, Li SY, Tu CC, Padma VV, Shih HN, Kuo WW and Huang CY: Thymoquinone suppresses migration of LoVo human colon cancer cells by reducing prostaglandin E2 induced COX-2 activation. World J Gastroenterol 23: 1171-1179, 2017.

4. Hossen MJ, Yang WS, Kim D, Aravinthan A, Kim JH and Cho JY: Thymoquinone: An IRAK1 inhibitor with in vivo and in vitro anti-inflammatory activities. Sci Rep 7: 42995, 2017. 
5. Cobourne-Duval MK, Taka E, Mendonca P, Bauer D and Soliman KF: The antioxidant effects of thymoquinone in activated BV-2 murine microglial cells. Neurochem Res 41: 3227-3238, 2016.

6. Ke X, Zhao Y, Lu X, Wang Z, Liu Y, Ren M, Lu G, Zhang D, Sun Z, Xu Z, et al: TQ inhibits hepatocellular carcinoma growth in vitro and in vivo via repression of Notch signaling. Oncotarget 6: 32610-32621, 2015.

7. Taha MM, Sheikh BY, Salim LZ, Mohan S, Khan A, Kamalidehghan B, Ahmadipour F and Abdelwahab SI: Thymoquinone induces apoptosis and increase ROS in ovarian cancer cell line. Cell Mol Biol (Noisy-le-grand) 62: 97-101, 2016

8. Yang J, Kuang XR, Lv PT and Yan XX: Thymoquinone inhibits proliferation and invasion of human nonsmall-cell lung cancer cells via ERK pathway. Tumour Biol 36: 259-269, 2015

9. Wilson AJ, Saskowski J, Barham W, Yull F and Khabele D: Thymoquinone enhances cisplatin-response through direct tumor effects in a syngeneic mouse model of ovarian cancer. J Ovarian Res 8: 46, 2015.

10. Park EJ, Chauhan AK, Min KJ, Park DC and Kwon TK: Thymoquinone induces apoptosis through downregulation of c-FLIP and Bcl-2 in renal carcinoma Caki cells. Oncol Rep 36: 2261-2267, 2016.

11. Jeghers H, Mckusick VA and Katz KH: Generalized intestinal polyposis and melanin spots of the oral mucosa, lips and digits; a syndrome of diagnostic significance. N Engl J Med 241: 1031-1036, 1949.

12. LKB1 and AMPK maintain epithelial cell polarity under energetic stress. J Cell Biol 203: 373, 2013.

13. Dahmani R, Just PA, Delay A, Canal F, Finzi L, Prip-Buus C, Lambert M, Sujobert P, Buchet-Poyau K, Miller E, et al: A novel LKB1 isoform enhances AMPK metabolic activity and displays oncogenic properties. Oncogene 34: 2337-2346, 2015.

14. Kan JY, Yen MC, Wang JY, Wu DC, Chiu YJ, Ho YW and Kuo PL: Nesfatin-1/Nucleobindin-2 enhances cell migration, invasion, and epithelial-mesenchymal transition via LKB1/AMPK/TORC1/ZEB1 pathways in colon cancer. Oncotarget 7: 31336-31349, 2016.

15. Taliaferro-Smith L, Nagalingam A, Zhong D, Zhou W, Saxena NK and Sharma D: LKB1 is required for adiponectin-mediated modulation of AMPK-S6K axis and inhibition of migration and invasion of breast cancer cells. Oncogene 28: 2621-2633, 2009.

16. Marcus AI and Zhou W: LKB1 regulated pathways in lung cancer invasion and metastasis. J Thorac Oncol 5: 1883-1886, 2010.

17. Li X, Teng S, Zhang Y, Zhang W, Zhang X, Xu K, Yao H, Yao J, Wang $\mathrm{H}$, Liang $\mathrm{X}$ and Hu Z: TROP2 promotes proliferation, migration and metastasis of gallbladder cancer cells by regulating PI3K/AKT pathway and inducing EMT. Oncotarget 8 : 47052-47063, 2017.

18. Li N,Huang D, Lu N and Luo L: Role of the LKB1/AMPK pathway in tumor invasion and metastasis of cancer cells (Review). Oncol Rep 34: 2821-2826, 2015.

19. Ahmad I, Muneer KM, Tamimi IA, Chang ME, Ata MO and Yusuf N: Thymoquinone suppresses metastasis of melanoma cells by inhibition of NLRP3 inflammasome. Toxicol Appl Pharmacol 270: 70-76, 2013.
20. Kolli-Bouhafs K, Boukhari A, Abusnina A, Velot E, Gies JP, Lugnier $\mathrm{C}$ and Rondé $\mathrm{P}$ : Thymoquinone reduces migration and invasion of human glioblastoma cells associated with FAK, MMP-2 and MMP-9 down-regulation. Invest New Drugs 30: 2121-2131, 2012

21. Zhou Y, Zhu Y, Fan X, Zhang C, Wang Y, Zhang L, Zhang H, Wen T, Zhang K, Huo X, et al: NID1, a new regulator of EMT required for metastasis and chemoresistance of ovarian cancer cells. Oncotarget 8: 33110-33121, 2017.

22. Zhao S, Sun H, Jiang W, Mi Y, Zhang D, Wen Y, Cheng D, Tang H, Wu S, Yu Y, et al: miR-4775 promotes colorectal cancer invasion and metastasis via the Smad7/TGF $\beta$-mediated epithelial to mesenchymal transition. Mol Cancer 16: 12, 2017.

23. Khan MA, Tania M, Wei C, Mei Z, Fu S, Cheng J, Xu J and Fu J: Thymoquinone inhibits cancer metastasis by downregulating TWIST1 expression to reduce epithelial to mesenchymal transition. Oncotarget 6: 19580-19591, 2015.

24. Weng J, Zhang H, Wang C, Liang J, Chen G, Li W, Tang H and Hou J: miR-373-3p targets DKK1 to promote EMT-induced metastasis via the $\mathrm{Wnt} / \beta$-catenin pathway in tongue squamous cell carcinoma. Biomed Res Int 2017: 6010926, 2017.

25. Chen J, Zhang H, Chen Y, Qiao G, Jiang W, Ni P, Liu X and Ma L: miR-598 inhibits metastasis in colorectal cancer by suppressing JAG1/Notch2 pathway stimulating EMT. Exp Cell Res 352: 104-112, 2017

26. Wei S, Wang L, Zhang L, Li B, Li Z, Zhang Q, Wang J, Chen L, Sun G, Li Q, et al: ZNF143 enhances metastasis of gastric cancer by promoting the process of EMT through PI3K/AKT signaling pathway. Tumour Biol 37: 12813-12821, 2016.

27. Li J, Liu J, Li P, Mao X, Li W, Yang J and Liu P: Loss of LKB1 disrupts breast epithelial cell polarity and promotes breast cancer metastasis and invasion. J Exp Clin Cancer Res 33: 70, 2014.

28. Liang X, Wang $\mathrm{P}$, Gao $\mathrm{Q}$ and Tao X: Exogenous activation of LKB1/AMPK signaling induces $\mathrm{G}(1)$ arrest in cells with endogenous LKB1 expression. Mol Med Rep 9: 1019-1024, 2014.

29. Luo L, Huang W, Tao R, Hu N, Xiao ZX and Luo Z: ATM and LKB1 dependent activation of AMPK sensitizes cancer cells to etoposide-induced apoptosis. Cancer Lett 328: 114-119, 2013.

30. Goodwin JM, Svensson RU, Lou HJ, Winslow MM, Turk BE and Shaw RJ: An AMPK-independent signaling pathway downstream of the LKB1 tumor suppressor controls Snaill and metastatic potential. Mol Cell 55: 436-450, 2014.

31. Roy BC,Kohno T, Iwakawa R, Moriguchi T,Kiyono T,Morishita K, Sanchez-Cespedes M, Akiyama T and Yokota J: Involvement of LKB1 in epithelial-mesenchymal transition (EMT) of human lung cancer cells. Lung Cancer 70: 136-145, 2010.

32. Kopsiaftis S, Sullivan KL, Garg I, Taylor JR III and Claffey KP: AMPK $\alpha 2$ regulates bladder cancer growth through SKP2-mediated degradation of p27. Mol Cancer Res 14: 1182-1194, 2016

33. Zhan P, Zhao S, Yan H, Yin C, Xiao Y, Wang Y, Ni R, Chen W, Wei $G$ and Zhang P: $\alpha$-enolase promotes tumorigenesis and metastasis via regulating AMPK/mTOR pathway in colorectal cancer. Mol Carcinog 56: 1427-1437, 2017. 